Bull. Mater. Sci., Vol. 14, No. 1, February 1991, pp. 87-91. (C) Printed in India.

\title{
Effect of annealing on crystal size in pure Mysore silk fibres
}

\author{
R SOMASHEKAR and R GOPALKRISHNA URS* \\ Department of Physics, University of Mysore, Manasagangotri, Mysore 570006 , India \\ *Department of Physics, National Institute of Engineering, Mysore 570 008, India \\ MS received 15 December 1990; revised 26 February 1991
}

\begin{abstract}
Wide angle X-ray diffraction studies of pure Mysore silk fibres, annealed at various temperatures at different periods of time were carried out to evaluate crystal size and lattice distortion parameters as these determine the properties of silk fibres.
\end{abstract}

Keywords. Crystal size; lattice distortion; Fourier co-eficients; pure Mysore silk fibre.

\section{Introduction}

The determination of crystallinity and disorder of fibres is important for textile industries, as they determine the properties of silk fibres. X-ray diffraction studies show that silk fibres are partially crystalline (Mathews 1951). X-ray scattering at wide angle $\left(\simeq 20^{\circ}\right)$ is due to the crystal lattice planes. For perfect crystalline lattice the crystal size can be obtained from the width of diffraction peaks using Scherrer's equation. However paracrystalline disorder of the lattice, which is also thought to exist in natural silk fibres also broadens the X-ray diffraction spots (Klug and Alexander 1954). In this paper we have attempted to determine the crystal size and lattice distortion parameters from the intensity profile of a fairly ciear (210) X-ray reflection obtained from annealed natural silk fibres at various temperatures and at various length of time. Such studies have not been reported earlier, except for determining the cell parameters (Marsh et al 1955; Shaw and Smith 1961; Warwicker 1961) and percentage of crystallinity (Bhat et al 1978) for natural silk fibre (Bombyx mori).

Both multiple and single-order methods used to separate crystal size and distortion parameters derive from the theory of Warren-Averbach (1950), utilizing the Fourier cosine coefficients of the intensity profile. Somashekar et al (1989) and Hall and Somashekar (1990) have considered various aspects of multiple and singleorder methods and suggested a suitable single-order method to obtain crystallite size and lattice distortion parameter from polymer fibres. Recently we have extended. this method to natural polymers (Somashekar et al 1990).

\section{Theory}

The intensity profile of the X-ray reflection from a partially crystalline sample like natural fibre is a function of the distribution of crystal sizes $\langle D\rangle$ and of the lattice distortion $(g)$ and these are related through the Fourier coefficients $A(n)$ to the profile intensity $I(s)$ by the equation

$$
I(s)=\sum_{n=-\infty}^{\infty} A(n) \cos \left\{2 \pi n d\left(s-s_{0}\right)\right\}
$$


Here $s_{0}$ is the value of $s=(\sin \theta / \lambda)$ at the peak of the profile and $d$ the mean $d$ spacing of lattice planes causing the reflection and $n$ the harmonic number.

Also the Fourier coefficients can be factorized into size $A_{s}(n)$ and disorder coefficients $A_{d}(n)$

$$
A(n)=A_{s}(n) \cdot A_{d}(n)
$$

These are not normalized. By taking exponential distribution function for crystal sizes, we have the following relations for $A_{s}(n)$

$$
\begin{aligned}
& A_{s}(n)=A(0)[1-n /\langle N\rangle] \quad n \leqslant p, \\
& A_{s}(n)=A(0)[\exp \{-\alpha(n-p)\} /\langle N\rangle], \quad n \geqslant p,
\end{aligned}
$$

where $\langle N\rangle$ is the average number of unit cells in a column through the crystal direction normal to the lattice planes causing reflection. Here $p$ is the smallest number of unit cells in a column.

The crystal size is given by

$$
D=\langle N\rangle d h k l
$$

and $A_{d}(n)$ is the disorder coefficient for paracrystal with separation of neighbouring lattice planes having Gaussian distribution of standard deviation given by

$$
A_{d}(n)=\exp \left(-2 \pi^{2} m^{2} n g^{2}\right)
$$

where $m$ is the order of reflection and $g$ the latice distortion parameter.

The reasons for using non-normalized Fourier coefficients are (i) truncation of the profile and (ii) error in the background estimation and these affect the low-order Fourier coefficients of the intensity profile as explained in detail by Somashekar et al (1989). We have therefore used asymmetric exponential distribution function for the crystal size distribution which gives fairly good results.

Using (1), (2), (4) and (5) along with experimental intensity data it is possible to obtain crystal size and lattice distortion.

\section{Experimental and computation}

Pure Mysore silk fibre is an indigenous multivoltine race of mulberry silk worm (Bombyx mori). The cocoons are kept in boiling water for about 2-3 min and fibre reeling is processed at $45^{\circ} \mathrm{C}$. Samples of silk fibres were annealed at 100,140 and $200^{\circ} \mathrm{C}$ for various length of time without stretching the fibres.

\subsection{X-ray diffraction pattern}

The X-ray diffraction profile of equatorial reflections from silk fibres, recorded using an X-ray diffractometer (JEOL, Japan) (target $\mathrm{Fe}, \lambda=1.934 \AA$ ) is given in figure 1 and has only two reflections. Of these, the (100) reflection has too much of background and we were not able to record a clear profile of the $(100)$ reflection, using an X-ray diffractometer. We have therefore used (210) reflection for our study. The reflections were identified using cell parameters reported earlier (Marsh et al 1955). The profile of (210) reflection used to obtain the crystal size and lattice 




Figure 1. X-ray diffractometer recording of pure Mysore silk fibre along equatorial direction.

distortion was assumed to be symmetric and the half where the overlap with the neighbouring reflection is minimum was used to determine the cosine Fourier coefficients $A(n)$. The background level was taken as that at which the intensity became uniform and this was subtracted from all the points. The scattering angle was transformed to $\sin (\theta) / \lambda$ and the Fourier coefficients were calculated from these intensity data after they were corrected for Lorentz and polarization factors.

In order to correct for instrumental line broadening using Stokes method (1948), $X$-ray diffraction pattern was recorded for aluminium foil under the same conditions as used for silk fibres. This procedure was repeated for all the samples annealed at various temperatures for various time periods.

\subsection{The refinement procedure}

The calculation of intensity profile using equations (1), (3) and (5) requires four parameters namely lattice distortion $g$, crystal size $(\langle N\rangle$ or $D=\langle N\rangle d h k l)$, error in the background and a parameter defining the width of the exponential distribution function of column lengths. Initial values of $g$ and $\langle N\rangle$ were obtained using the method of Nandi et al (1984). Using these values in the above mentioned equations gave the corresponding values for the distribution width. These are only rough estimates but the refinement procedure must be sufficiently robust to start with such inaccurate values. Here we compute

$$
\Delta^{2}=\left[I_{\text {cal }}-\left(I_{\exp }+B G\right)\right]^{2} / \text { number of points. }
$$

The value of $\Delta$ was divided by half the maximum value of intensity so that it is expressed relative to the mean value of intensities and this function is minimized. For refinement, the multidimensional minimization algorithm of SIMPLEX method was used (Press et al 1986). Here $B G$ refers to inaccuracy in background estimation.

It was observed that the variation of $\langle N\rangle, p$ and $\alpha$ defined in (3) with respect to $g$ for exponential distribution function are almost constant and under these 
circumstances, the average values of parameters $\langle N\rangle, p$ and $\alpha$ were used to determine the $g$ value and these are given in table 1.

All the necessary computer programs were written in FTN77 language and were compiled and executed using Archimedes $310 \mathrm{M}$, Acorn (UK make).

\section{Results and discussion}

Table 1 gives the parameters needed for recalculating the intensity profile, using the equations mentioned earlier in the text. Figure 2 shows good agreement between experimental and the intensity calculated on the basis of the paracrystalline model suggested in this paper for (210) reflection in silk fibre annealed at different

Table 1. Paracrystalline parameters obtained from (210) X-ray reflection by silk fibres.

\begin{tabular}{|c|c|c|c|c|c|c|c|}
\hline Fibre & $\langle N\rangle$ & $p$ & $\bar{\alpha}$ & $g$ & $A(0)$ & $B G$ & $D($ in $\AA)$ \\
\hline Pure Mysore silk (PMS) & $2.85 \pm 0.14$ & $0.58 \pm 0.27$ & $0.42 \pm 0.02$ & $5.7 \%$ & $50 \cdot 6$ & 1.0 & 12.05 \\
\hline PMS annealed at $100^{\circ} \mathrm{C} 7 \mathrm{~h}$ & $3.40 \pm 0.21$ & $0.80 \pm 0.05$ & $0.38 \pm 0.03$ & $2.8 \%$ & $30-6$ & 1.0 & $14 \cdot 38$ \\
\hline PMS annealed at $100^{\circ} \mathrm{C} 14 \mathrm{~h}$ & $3.49 \pm 0.15$ & $1 \cdot 26 \pm 0.07$ & $0.45 \pm 0.02$ & $9 \cdot 2 \%$ & $42 \cdot 0$ & -0.06 & $14 \cdot 76$ \\
\hline PMS annealed at $140^{\circ} \mathrm{C} 7 \mathrm{~h}$ & $4.58 \pm 0.29$ & $2 \cdot 20 \pm 002$ & $0.42 \pm 0.02$ & $7.5 \%$ & $33 \cdot 9$ & -0.85 & $19 \cdot 37$ \\
\hline PMS annealed at $200^{\circ} \mathrm{C} 7 \mathrm{~h}$ & 3.65 & $0-58$ & 0.33 & $3-0 \%$ & $26 \cdot 2$ & -0.55 & $15 \cdot 43$ \\
\hline
\end{tabular}

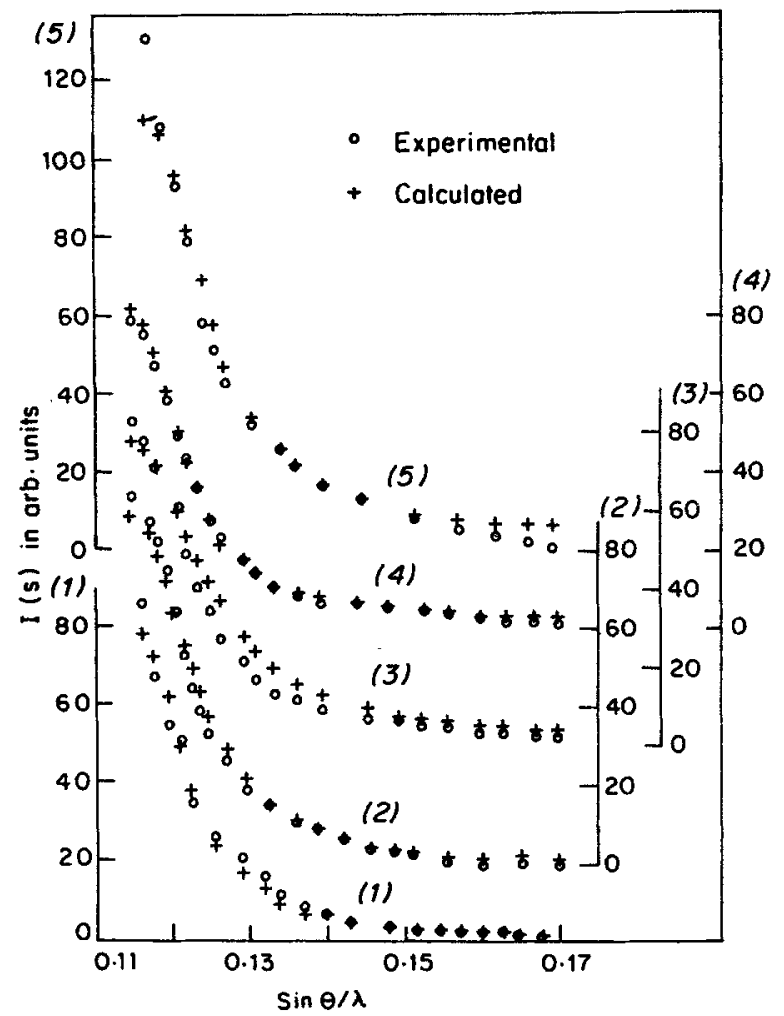

Figure 2. Experimental and calculated intensity of (210) $X$-ray reflection by silk fibres annealed at various temperatures for various length of time. (1) $200^{\circ} \mathrm{C} 7 \mathrm{~h}$, (2) $140^{\circ} \mathrm{C} 7 \mathrm{~h}$, (3) $100^{\circ} \mathrm{C} 14 \mathrm{~h},(4) 100^{\circ} \mathrm{C} 7 \mathrm{~h}$ and (5) native pure silk fibre. 
temperatures. This clearly indicates that the parameters obtained here are quite reliable. The reliability of this procedure has been investigated (Hall and Somashekar 1990) by comparing with the existing multiple order method of Warren and Averbach (1950). The $D$ values shown in table 1 increase with increase in annealing temperatures as well as with the increase in length of time. This is because at higher temperatures, the protein molecules (in this case $\beta$-pleated structure) may arrange themselves such that when frozen may result in increase of crystalline region; in other words, the number of unit cell increases with annealing temperature. The decrease above $150^{\circ} \mathrm{C}$ is due to the marked transition that occurs both internally and externally and which is seen in the form of total decoloration (brownish red) of silk fibres but still retaining all the features of fibres. Variation of $g$ with annealing temperature which is about $3-9 \%$ gives the extent of lattice distortion in silk fibres along [210] direction and to some degree depends on the model used to separate the crystal size and distortion coefficients from the Fourier coefficients. It is well known that the strength of fibres increases with increase in crystal size in the case of man-made fibres and hence we can infer that the strength of silk fibres can be improved by annealing the fibres.

\section{Conclusion}

The crystal size in natural silk fibre is less compared to annealed silk fibre, is maximum in the fibre annealed at $140^{\circ} \mathrm{C}$ for $7 \mathrm{~h}$ and decreases above this temperature. Also, the variation of distortion parameter at different temperatures is small. This study shows that one can improve the strength of the pure Mysore silk fibres by annealing at $140^{\circ} \mathrm{C}$ for $7 \mathrm{~h}$ without loss of lustre of the silk fibres.

\section{Acknowledgement}

The authors thank the Director, Central Sericulture Research and Training Institute, Mysore, for providing samples and Dr I H Hall, UMIST, Manchester, England, for updating the information with regard to single-order method.

\section{References}

Bhat N V, Nadiger G T and Venkatraman A 1978 Proc. Nucl. Phys. Solid State Phys. C21 431

Hall I H and Somashekar R $1990 \mathrm{~J}$. Appl. Crystallogr. (Communicated)

Klug H P and Alexander L E 1954 X-ray diffraction procedures (New York: John Wiley)

Marsh R E, Corey R B and Pauling L 1955 Biochim. Biophys. Acta. 161

Mathews J M 1951 Textile fibres (New York: John Wiley)

Nandi R K, Kuo H R, Schlosberg M, Wissler G, Cohen J B and Crist B J 1984 J. Appl. Crystallogr. 17 22

Press W, Flannery B P, Tenkolsky S and Vettering W T 1986 Numerical recipes (Cambridge: University Press) p 294 and 289

Shaw J T B and Smith S G 1961 Biochim. Biophys. Acta 52305

Somashekar R, Hall I H and Carr P D 1989 J. Appl. Crystallogr. 22363

Somashekar R, Gopalkrishna Urs R, Sing R S and Ali G K 1990 Starch/Starck (Communicated)

Stokes A R 1948 Proc. Phys. Soc. London 61382

Warren B E and Averbach B L 1950 J. Appl. Phys. 21595

Warwicker J O 1961 Biochim. Biophys. Acta 52319 